\title{
ESTREPTOQUINASE E OXIGÊNIO HIPERBÁRICO EM CONGESTÃO APÓS REIMPLANTE DE MEMBRO
}

\author{
Marcelo Sacramento Cunha, José Carlos faes da Silva*, Hugo Alberto Nakamoto, Klaus Werner fels, Marcus Castro Ferreira \\ Trabalho realizado na Disciplina de Cirurgia Plástica da Faculdade de Medicina da Universidade de São Paulo, Laboratório de Cirurgia Plástica e \\ Microcirurgia da FMUSP.
}

*Correspondência:

Av. Jacutinga, 635, apto 12

São Paulo/SP

Cep: $04515-030$

zefaes@osite.com.br

\section{RESUMO}

Oвjetivo. Foram testados os efeitos do fármaco estreptoquinase e da terapia com oxigênio hiperbárico em modelo experimental de oclusão venosa após reimplante de membro.

Métodos. Foram realizadas amputações com preservação de vasos e nervos dos membros posteriores direitos de 140 ratos. Os grupos GM0, GMI, GM2, GM3 e GM4 foram submetidos a tempos de oclusão venosa de zero, uma, duas, três e quatro horas. Os grupos GEI e GE2 foram tratados com estreptoquinase e terapia com oxigênio hiperbárico, respectivamente, após oclusão venosa de três horas. Os resultados foram analisados estatisticamente pelo teste do Qui-quadrado $(p<0,05)$.

Resultados. As taxas de mortalidade transoperatórias dos grupos GM0, GMI, GM2, GM3 e GM4 foram 0\%, 10\%, 15\%, $30 \%$ e $60 \%$ e as pós-operatórias foram $5 \% ; 11,1 \% ; 11,7 \% ; 14,2 \%$ e 100\%, respectivamente. As taxas de viabilidade dos membros isquêmicos após sete dias de avaliação foram 100\%, 87,5\%,80\% e 66,67\%. As taxas de viabilidade dos grupos GEI e GE2 foram 76,9\% e 100\%, respectivamente. As taxas de mortalidade transoperatórias foram diferentes estatisticamente com exceção de GMI e GM2. As taxas de mortalidade pós-operatórias não foram diferentes com exceção de GM3 e GM4. As taxas de viabilidade dos grupos modelo foram diferentes entre si, exceto os grupos GMI e GM2. GEI resultou em uma viabilidade de membros sem diferença estatística e GE2 em uma viabilidade de membros maior que GM3.

Conclusã̃o. A estreptoquinase não alterou os efeitos da oclusão venosa e a terapia com oxigênio hiperbárico aumentou a viabilidade dos membros.

Unitermos: Ratos Wistar. Membro. Oclusão venosa. Estreptoquinase. Oxigênio hiperbárico.

\section{INTRODUÇÃO}

A oclusão venosa em retalhos pediculados, microcirúrgicos e reimplantes continua sendo um problema significativo para o cirurgião. A insuficiência venosa é a causa mais comum de perda de reimplantes digitais'. A oclusão venosa leva a um aumento na pressão hidrostática na microcirculação, levando à lesão endotelial com extravasamento de plasma e células. O edema formado aumenta a pressão intersticial com compressão externa dos vasos e isquemia secundária. Observa-se depósitos de fibrina e ativação e agregação plaquetária após lesão endotelial, levando à trombose da microcirculação. Os neutrófilos são ativados e produzem substâncias tóxicas, entre elas os radicais livres do oxigênio ${ }^{2}$. A lesão tecidual após a oclusão venosa é progressiva, com reperfusão lenta do membro, manutenção da isquemia secundária, progressão da trombose da microcirculação e morte tecidual ${ }^{2}$.

Trabalhos relatam que a oclusão venosa pós-operatória é mais deletéria que a oclusão global ou arterial. Pequenos períodos de oclusão venosa podem levar a efeitos pronunciados e de longa duração na microcirculação, levando ao comprometimento da viabilidade de retalhos e membros ${ }^{3,4.5}$. Estudos experimentais confirmaram que retalhos de pele podem suportar tempos de oclusão arterial ou arterial e venosa de oito horas, mas a oclusão venosa pelo mesmo período leva à necrose total dos retalhos ${ }^{6}$. O músculo esquelético é mais sensível à oclusão venosa que a pele. Harashima et al. ${ }^{7}$ observaram 100\% de necrose em retalhos de pele em ratos após oito horas de oclusão venosa enquanto Gabriel et al. ${ }^{3}$ observaram lesões teciduais irreversíveis após períodos de 60 minutos em retalho de músculo grácil.

Diversos modelos experimentais de retalho cutâneo e musculares têm sido desenvolvidos em ratos e porcos para o estudo da fisiopatologia da oclusão venosa. Poucos trabalhos estudaram os efeitos da oclusão venosa em tecidos compostos e membros.

A fisiopatologia da congestão venosa envolve estase, hemoconcentração, fluxo lento, ativaçãa da cascata de coagulação, concentração de plaquetas e fibrinogênio. Considerando estas alterações no tecido congesto, existem justificativas teóricas para a utilização de fibrinolíticos. A estreptoquinase e 0 ativador do plasminogênio tecidual têm sido efetivos em salvar retalhos livres em estudos clínicos e experimentais ${ }^{8,9}$.

A terapia com oxigênio hiperbárico tem sido aplicada em uma série de situações clínicas. A utilização da terapia com oxigênio hiperbárico em retalhos isquêmicos, enxertos compostos e na preservação de membros antes do reimplante tem sido estudada. Os resultados para retalhos isquêmicos são conflitantes e muito animadores para enxertos compostos e preservação de membros isquêmicos ${ }^{10-16}$. Alguns trabaIhos relatam efeitos benéficos da terapia com oxigênio hiperbárico em retalhos cutâneos experimentais submetidos à oclusão venosa ${ }^{17-19}$. Os 
CUNHA MS ET AL.

efeitos da terapia com oxigênio hiperbárico em tecidos compostos ou membros reimplantados e submetidos a oclusão venosa ainda não foram esclarecidos.

O presente trabalho pretendeu testar o efeito da administração do fármaco trombolítico estreptoquinase e da terapia com oxigênio hiperbárico em um modelo de oclusão venosa após reimplante de membro em ratos.

\section{Métodos}

Foram utilizados I 40 ratos Wistar machos, pesando entre $290 \mathrm{~g} \mathrm{e}$ 310 g, ração e água ad libitum e período claro/escuro de 12/12 horas.

Os animais foram anestesiados com tiopental sódico $(50 \mathrm{mg} / \mathrm{Kg})$ intraperitonial. Incisão vertical da pele e tecido celular subcutâneo em região inguinal direita foi realizada com identificação dos vasos femorais e seus ramos. Realizou-se incisão circular na porção média da coxa abrangendo pele, tecido celular subcutâneo e musculatura com prévia eletrocauterização, mantendo o nervo ciático identificado e intacto. A osteotomia do fêmur no terço médio foi realizada com serra manual, utilizando um segmento de látex para proteção dos vasos e nervos do membro. Obteve-se uma amputação do membro posterior direito com preservação de vasos e membros. Procedeu-se a síntese óssea com fixação intramedular do fêmur com segmento de $2 \mathrm{~cm}$ de agulha $30 \times 7$ e cola de éster de cianoacrilato e na síntese da musculatura foram utilizados pontos de nálon 5.0. Aplicou-se o clipe microvascular atraumático na veia femoral antes da emergência dos vasos epigástricos superficiais caudais, evitando-se incluir o nervo e artéria femorais. Observou-se a interrupção do fluxo sangüíneo da veia femoral por meio do teste de ordenhamento negativo. $\mathrm{Na}$ síntese da pele, foi utilizada sutura contínua de náilon 5.0. A reposição volêmica foi realizada por meio da injeção subcutânea de $50 \mathrm{ml} / \mathrm{Kg}$ de solução fisiológica de $\mathrm{NaCl}$ a $0,9 \%$, após o procedimento cirúrgico.

Os animais foram submetidos a tempos de oclusão venosa quente a partir do pinçamento dos vasos. Durante esse período, os animais foram mantidos em gaiolas individuais a uma temperatura ambiente de $25^{\circ} \mathrm{C}$. Após o período de oclusão venosa, os ratos foram anestesiados com tiopental sódico e realizou-se incisão vertical em região inguinal esquerda com dissecção e canulação da veia epigástrica superficial caudal com agulha 29G. A sutura vertical da pele do membro posterior direito foi aberta e retirado o clipe vascular. Antes da retirada do clipe vascular, foram injetadas soluções (soro fisiológico ou estreptoquinase) com volume total de I ml na veia epigástrica superficial caudal esquerda nos grupos modelos e experimentais. Procedeu-se, então, a síntese da pele das incisões e os animais foram acondicionados em gaiolas individuais.

Os animais foram divididos em grupos modelos e experimentais. Os cinco grupos modelos, com 20 ratos cada, foram submetidos a tempos de oclusão venosa de zero, uma, duas, três e quatro horas (GM0, GMI, GM2, GM3, GM4, respectivamente).

O GM3 foi utilizado como controle dos grupos experimentais que foram assim divididos:

I. Grupo experimental I (GEI): 20 ratos foram submetidos ao procedimento cirúrgico, três horas de oclusão venosa e foi injetado I ml de solução contendo $20.000 \mathrm{UI} / \mathrm{Kg}$ de estreptoquinase em bolus ${ }^{20,21}$.
2. Grupo experimental 2 (GE2): 20 ratos foram submetidos ao procedimento cirúrgico, três horas de oclusão venosa e foi injetado I ml de solução salina $0,9 \%$ em bolus. Os ratos foram levados à câmara hiperbárica modelo Seecrhist até uma hora após o fim da oclusão venosa. Foram realizadas seis sessões de 30 minutos com oxigênio a $100 \%$ a 2,5 ATM, períodos de compressão e descompressão de cinco minutos e intervalos de 12 horas entre as sessões ${ }^{21}$.

Os animais foram observados diariamente por um período de sete dias. Os óbitos observados foram divididos de acordo com o período em que ocorreram: período transoperatório e período pós-operatório. A mortalidade transoperatória incluiu os óbitos ocorridos durante o procedimento cirúrgico desde a aplicação da anestesia até a liberação do clipe vascular. A mortalidade pós-operatória incluiu os óbitos ocorridos desde a liberação do clipe vascular até o sétimo dia de pós-operatório.

A viabilidade dos membros foi determinada por meio de exame clínico e os membros foram classificados em viáveis ou não viáveis. $O$ membro não viável foi definido pela necrose total do membro. O viável foi definido por sobrevivência total do membro ou sobrevivência total e presença de necrose parcial apenas de pele próxima à sutura com presença de músculo viável subjacente e crescimento de pêlos.

Um rato foi excluído do GE2 por problema relacionado ao clipe vascular (acidentalmente mantido no pedículo) e os ratos que evoluíram para óbito no período transoperatório foram excluídos após o cálculo da taxa de mortalidade transoperatória para tornar possível o cálculo da taxa de mortalidade pós-operatória. Os ratos que evoluíram para óbito no período pós-operatório foram excluídos após o cálculo da taxa de mortalidade pós-operatória para permitir o cálculo da taxa de viabilidade dos membros dos ratos vivos após sete dias. Os animais foram sacrificados com superdose de éter inalatório.

Os resultados foram transformados em taxas (porcentagens) de mortalidade dos ratos e viabilidade do membro por grupo. Os grupos foram comparados entre si e analisados estatisticamente pelo teste do Qui-quadrado. O nível de significância adotado foi de $5 \%(p<0,05)$.

\section{Resultados}

Quarenta e sete animais evoluíram para óbito, durante o procedimento cirúrgico (27 animais) ou até o sétimo dia de pós operatório (20 animais), levando a uma mortalidade total de 33,8\%. No período pós-operatório, os grupos GM3 e GM4 evoluíram com taxas de mortalidade diferentes estatisticamente $(p<0,00 \mathrm{I})$. As taxas de mortalidade no período transoperatório foram diferentes significativamente entre os grupos GM0 e GMI $(p<0,005)$, GM2 e GM3 $(p<0,005)$ e GM3 e GM4 ( $p<0,00 I)$.

A viabilidade do membro encontrada após sete dias de observação foi do tipo tudo ou nada e está representada no Gráfico 2.

Os grupos GM0 e GMI $(p<0,00 I)$, GM2 e GM3 $(p<0,05)$ foram diferentes estatisticamente. Os grupos GMI e GM2 não foram diferentes estatisticamente $(p<0,5 \%)$.

O GEI evoluiu com taxa de viabilidade dos membros de $76,9 \%$ e não foi diferente estatisticamente do grupo GM3 $(p<0, I)$. O GE2 evoluiu com taxa de viabilidade dos membros de $100 \%$ e foi diferente estatisticamente do grupo GM4 $(p<0,00$ I). 

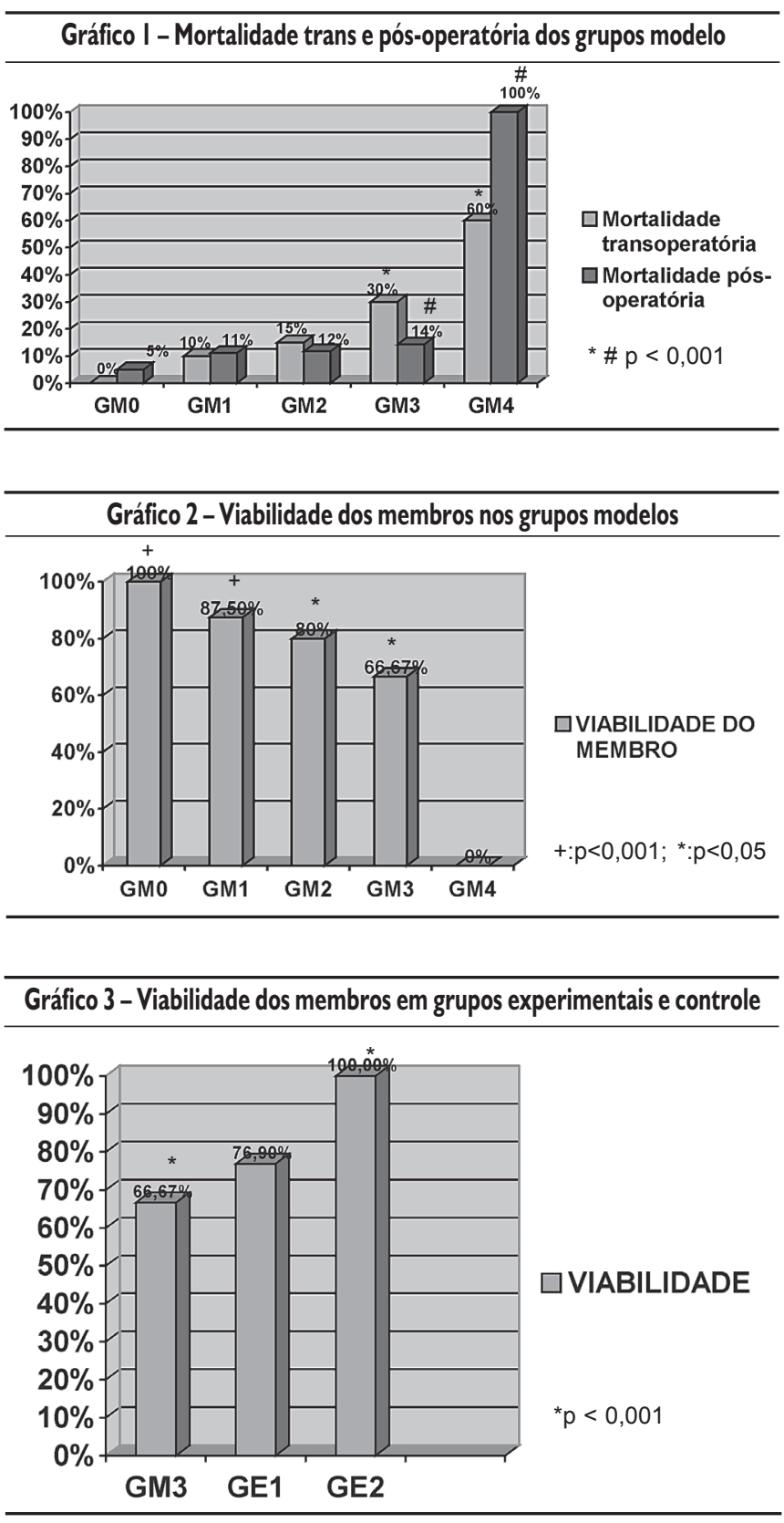

\section{Discussão}

Vários modelos de retalhos têm sido propostos para o estudo da lesão tecidual por oclusão venosa. Harashina et al. ${ }^{7}$, em 1977, foram os primeiros autores a desenvolverem um modelo de oclusão venosa em retalhos de pele em ratos. Daniel et al. ${ }^{24}$, em 1982, desenvolveram um modelo de oclusão venosa em retalhos cutâneos de porcos que foi posteriormente utilizado por Hedén et al. ${ }^{5}$ para estudo dos eventos circulatórios e metabólicos da oclusão venosa. Ao final da década de 80, com o interesse pelo estudo da congestão, em particular seus efeitos sobre o músculo, foram desenvolvidos novos modelos de retalhos miocutâneos $3,8,25,26$. No final da década de 90 , surgiu o interesse pelos efeitos da oclusão venosa em retalhos compostos e membros.
Nakamura et al. ${ }^{2}$, em 1998, desenvolveram um modelo de reimplante de membro posterior de coelhos com preservação de vasos em que era procedido o clipamento da veia femoral. $\mathrm{O}$ presente trabalho utilizou um modelo de oclusão venosa após reimplante de membro posterior de ratos. O procedimento cirúrgico constou de amputação parcial mantendo o pedículo vascular e nervoso do membro semelhante ao modelo utilizado por Concannon et al. ${ }^{27}$ e Cunha et al..$^{21-23}$ para estudos de isquemia.

A mortalidade foi estudada em dois momentos. A mortalidade transoperatória reflete os óbitos secundários às complicações anestésicas e efeitos sistêmicos da congestão venosa. As taxas de mortalidade transoperatória foram progressivas e estatisticamente significantes entre si, com exceção de GM0 e GMI. Considerando que as complicações anestésicas puras são iguais para todos os grupos, observa-se um gradual aumento dos efeitos deletérios da congestão por meio de uma curva tempo de oclusão venosa e mortalidade. O grupo GM4 destacou-se pela grande diferença estatística do grupo GM3, traduzida pela significância estatística $(p<0,00 \mathrm{I})$. A mortalidade pós-operatória reflete os óbitos secundários à reperfusão do membro e infecção maciça. As taxas de mortalidade pós-operatória foram significantes apenas entre os grupos GM3 e GM4. Os resultados sugerem que os efeitos sistêmicos da reperfusão do membro não são importantes até o tempo de três horas (tempo crítico) e se tornam importantes no tempo de quatro horas em que as lesões se tornam progressivas e irreversíveis.

A viabilidade dos membros mostrou-se como resposta do tipo tudo ou nada, como relatada em modelos de reimplantes isquêmicos de membro ${ }^{21-23,27}$. A redução gradual e significante das taxas de viabilidade ao longo dos grupos sugere uma curva tempo de oclusão venosa e viabilidade dos membros, tornando o modelo de oclusão venosa confiável. O grupo GM3 foi adotado como controle para o estudo da eficácia da estreptoquinase e terapia com oxigênio hiperbárico. Este grupo representou o maior tempo de oclusão venosa antes do tempo (GM4), em que a taxa de mortalidade pós-operatória foi de 100\%.

Estreptoquinase é um fármaco fibrinolítico ativador indireto do plasminogênio. Ativa o sistema fibrinolítico como um pró-ativador que converte o plasminogênio em plasmina, protease capaz de hidrolizar a plasmina e lisar o coágul ${ }^{28,29}$. Urokinase, heparina e prostaglandina EI fizeram parte do protocolo de reimplante de Fukui e Tamai ${ }^{30}$. Vertos e Tsavissis ${ }^{31}$ usaram estreptoquinase e dextran de baixo peso molecular rotineiramente após reimplantes. A estreptoquinase e rt-PA foram utilizadas com sucesso em relatos clínicos de reimplantes e modelos experimentais de trombose e reimplantes isquêmicos ${ }^{21,29,32}$.

No presente trabalho, o grupo GEI recebeu intravenosamente o fármaco estreptoquinase na dose de $20.000 \mathrm{UI} / \mathrm{Kg}$ após período de oclusão venosa de três horas. Esta dose foi escolhida com base em doses utilizadas para fibrinólise sistêmica na prática clínica. Esta dose também foi utilizada por Busnardo et al. ${ }^{20}$, em 1997, para estudo da trombose em anastomoses microcirúrgicas em ratos e por Cunha et al. ${ }^{21}$, em 2005, para o estudo de reimplante de membro isquêmico em ratos. A taxa de viabilidade dos membros isquêmicos foi de $76,9 \%$ e não foi diferente significativamente do grupo controle GM3. Este resultado sugere que a utilização da estreptoquinase não é suficiente para alterar a sobrevivência de membros congestos. A utilização de 
trombolíticos pode reduzir a coagulação intravascular e aumentar o fluxo de reperfusão, diminuindo a reperfusão lenta da congestão venosa. No entanto, poderia também aumentar a formação de hematomas intersticiais, aumentando a pressão intersticial e levando a uma piora na reperfusão. Estes dois mecanismos parecem atuar simultaneamente impedindo um efeito benéfico puro da estreptoquinase.

Existem várias hipóteses para o mecanismo de ação da terapia com oxigênio hiperbárico ${ }^{16}$. Manson et al. ${ }^{\prime \prime}$ e Zamboni et al. ${ }^{12}$ relataram o efeito benéfico do oxigênio hiperbárico em retalhos isquêmicos com neoformação de capilares. A proliferação de capilares no retalho foi observada por Ramon et al. ${ }^{13}$ através de microangiografia e este efeito poderia ser secundário à vasoconstricção que o oxigênio hiperbárico promovia na artéria epigástrica, o que estimularia a formação de novos capilares no retalho. Outro mecanismo de ação seria o seqüestro de neutrófilos para o tecido pulmonar. Os neutrófilos são fonte conhecida de radicais livres do oxigênio e atuam como mediadores de eventos biológicos que levam à lesão tecidual durante a reperfusão $0^{33}$. A terapia com oxigênio hiperbárico aumenta o oxigênio dissolvido no plasma e na presença de circulação sangüínea; isto resulta em aumento da tensão tecidual de oxigênio. À inalação de oxigênio a 100\% a 2,5 ATM de pressão, a tensão tecidual de oxigênio aumenta 12 vezes e a distância de difusão efetiva do oxigênio nos tecidos aumenta três vezes ${ }^{34}$. O oxigênio hiperbárico reduz o edema pós-traumático e lesões causadas pelos radicais livres do oxigênio em estudos experimentais com indícios de aumento da atividade da superóxido dismutase ${ }^{18}$.

Estudos experimentais têm mostrado efeitos benéficos da terapia com oxigênio hiperbárico em modelos de retalhos cutâneos submetidos a oclusão venosa ${ }^{18,19,35}$. Lozano et al. ${ }^{17}$ observaram que a terapia com oxigênio hiperbárico associada à utilização de sanguessugas diminui a área de necrose em retalhos epigástricos de pele em ratos após oclusão venosa total, porém somente o oxigênio hiperbárico não era benéfico.

O efeito da terapia com oxigênio hiperbárico em tecidos compostos ou membros foi pouco estudado. No presente estudo, foram utilizadas seis sessões de terapia com oxigênio hiperbárico nas primeiras 72 horas do período pós-operatório, com intervalo de 12 horas entre as sessões. Este mesmo esquema de sessões também foi utilizado por Prada ${ }^{10}$, em 1998, e Cunha et al. ${ }^{22}$, em 2005, para estudos de isquemia. A taxa de viabilidade dos membros submetidos a oclusão venosa por três horas e submetidos a terapia com oxigênio hiperbárico (GE2) foi significativamente maior que a taxa encontrada para o grupo controle GM3. Este resultado sugere um efeito benéfico na taxa de viabilidade dos membros submetidos a oclusão venosa.

Não foram encontrados trabalhos na literatura que tivessem utilizado membros submetidos a oclusão venosa e terapia com oxigennio hiperbárico. Vários mecanismos podem ser propostos para explicar o efeito benéfico. Na fisiopatologia da oclusão venosa estão envolvidos edema tecidual, produção de radicais livres do oxigênio, microtromboses da microcirculação e queda da tensão tecidual do oxigênio. A terapia com oxigênio hiperbárico mostrou-se eficaz experimentalmente em reduzir o edema tecidual, a produção de radicais livres do oxigênio por meio do aumento da função da enzima superóxido dismutase e em aumentar a tensão tecidual do oxigênio.

\section{ConClusão}

O fármaco estreptoquinase não mostrou benefício na taxa de viabilidade de membros submetidos a oclusão venosa após três horas. A evolução foi semelhante ao grupo controle.

A terapia com oxigênio hiperbárico levou a uma melhora na taxa de viabilidade de membros submetidos a oclusão venosa por três horas de $66,67 \%$ para $100 \%$.

\section{Conflito de interesse: não há.}

\section{SUMMARY}

EFFECTS OF STREPTOKINASE AND HYPERBARIC OXYGEN THERAPY ON VENOUS OCCLUSION AFTER LIMB REIMPLANTATION IN RATS

OBIECTIVE. The effectiveness of streptokinase and hyperbaric oxygen therapy on venous occlusion after limb reimplantation was tested in rats.

METHODS. Amputation with preservation of vessels and nerves of the right hind limb was carried out in 140 rats. Groups MGO, MG I, MG2, MG3 and MG4 were submitted to 0, 1, 2, 3 and 4 hours of venous occlusion. MG3 was elected as controlfor the experimental groups. Groups EGI and EG2 were submitted to 3 hours of venous occlusion and were treated with streptokinase and hyperbaric oxygen therapy. Limbs were observed for 7 days and their mortality and survival rates were studied.

RESULTS. Trans-operatorymortality rates in groups MGO, MGI, MG2, MG3 and MG4 were 0, 10, 15, 30 and 60\% respectively and the postoperatory mortality rates were $5 ; 11.1 ; 11.7 ; 14.2$ and $100 \%$ respectively. The limb survival rates were 100\%,87.5\%,80\% and 66.67\% respectively and $76.9 \%$ and $100 \%$ in EGI and EG2. Model groups were statistically different, except for MGI and MG2 in transoperatory mortality rates. There were no statistical differences in postoperatory mortality rates between model groups exceptfor MG3 and MG4. Modelgroups were statistically different, with the exception of MG I and MG2, in limb survival rates. EGI and MG3 showed no statistical difference in limb survival and EG2 had a better limb survival than MG3.

CONCLUSION. Results suggest that the administration of streptokinase does not change effects of venous occlusion and that hyperbaric oxygen therapy may decrease the effects of venous occlusion in limbs. [RevAssoc Med Bras 2007; 53(I): 29-33]

KEY WORDS: Rat limb. Venous occlusion. Streptokinase. Hyperbaric oxygen.

\section{REFERÊNCIAS}

I. Nichter LS, Haines PC, Edgerton MT. Successful replantation in the face of absent venous drainage: na experimental study. Plast Reconstr Surg. | 985;75:686-9|.

2. Nakamura K, Yokoyama K, Kimura M. Time course of superoxide generation in reperfusion after arterial ischemia and venous congestion. $\mathrm{Br}$ J Plast Surg. 1998;51:633-6.

3. Gabriel A, Chaney N, Stephenson LL, ZamboniWA. Effect of total venous oclusion on capillary flow and necrosis in skeletal muscle. Plast Reconstr Surg. 200 I; I 08:430-3.

4. Kerrigan CL, Wizman P, Hjortdal VE. Global flap ischemia: a comparison of arterial versus venous etiology. Plast Reconstr Surg. 1994;93: I 485-90.

5. Hedén P, Sollevi A. Circulatory and metabolic events in pig island skin flaps after arterial or venous oclusion. Plast Reconstr Surg. 1989;84:475-81. 
6. Su CT, Im MJ, Hoopes JE. Tissue glucose and lactate following vascular occlusion in island skin flaps. Plast Reconstr Surg. 1982;70:202-5.

7. Harashima T, Sawada Y, Watanebe S. The relationship between venous occlusion time in island skin flaps and flap survivals. Plast Reconstr Surg. 1977;60:92-5.

8. Hjortdal VE, Sinclair T, Kerrigan CL. Venous ischemia in skin flaps: microcirculatory intravascular thombosis. Plast Reconstr Surg. 1994:93:366-70.

9. Lantieri LA, Ozbek MR, Deune EG, Ornberg RL, Brown DM, Chung SH, et al. Prevention of microvascular thrombosis by topical application of recombinant tissue factor pathway inhibitor. Plast Reconstr Surg. 1996;97:587-94.

10. Prada FS. Efeito do alopurinol, da superóxido dismutase e da terapia com oxigênio hiperbárico em retalhos fásciocutâneos pediculados submetidos à oclusão temporária do pedículo vascular. Estudo experimental em ratos sobre isquemia e reperfusão [dissertação]. São Paulo: Faculdade de Medicina, Universidade de São Paulo; 1998.

I I. Manson PN, Im MJ, Myers RM. Improved capillaries by hyperbaric oxigen in skin flaps. Surg Forum. 1980; 31:564-8.

12. Zamboni WA, Roth AC, Russel RC, Nemiroff PM, Casas L, Smoot EC. The effect of acute hyperbaric oxigen therapy on axial pattern skin flap survival when administred during and after total ischemia. J Reconstr Microsurg. 1988;5:344-8.

13. Ramon Y, Abramovich A, ShupakA, Ullmann Y, Moscona RA, Shoshani O. Effect of hyperbaric oxigen on a rat transverse rectus abdominis myocutaneous flap model. Plast Reconstr Surg. 1998; 1 02:41 6-22.

14. Caffee HH, Galagher TJ. Experiments on the effects of hyperbaric oxigen on flap survival in the pig. Plast Reconstr Surg. 1988;81:751-8.

15. Zemmel NJ, Amis LR, Sheppard FR. A temporal analysis of the effects of pressurized oxigen on the $\mathrm{pH}$ of amputated muscle tissue. Ann Plast Surg. 1998;40:624-9.

16. Zhang F, Cheng C, Gerlach T. Effect of hyperbaric oxigen on survival of the composite ear graft in rats. Ann Plast Surg. 1998;41:530-4.

17. Lozano DD, Stephenson LL, Zamboni WA. Effect of hyperbaric oxygen and medicinal leeching on survival of axial skin flaps subjected to total venous occlusion. Plast Reconstr Surg. 1999; 104: 1029-32.

18. Yücel A, Bayramiçli M. Effects of hyperbaric oxygen treatment and heparin on the survival of unipedicled venous flaps: na experimental study in rats. Ann Plast Surg. 2000;44:295-9.

19. Gampper TJ, Zhang F, Mofakhami NF,Morgan RF, Amiss R, Hoard MA. Beneficial effect of hyperbaric oxygen on island flaps subjected to secondary venous ischemia. Microsurgery. 2002;22:49-52.

20. Busnardo FF, MunhozAM, Cunha M. Eficácia da estreptoquinase sistêmica na taxa de patência vascular em um modelo de trombose arterial em ratos. In: XXXIV Congresso Brasileiro de Cirurgia Plástica. São Paulo; 1997. Anais.

21. Cunha MS, da Silva JCF, Nakamoto H, Ferreira MC. Study of warm ischemia followed by reperfusion on a lower limb model in rats: effect of allopurinol and streptokinase. Clinics. 2005;60:2 I3-20.
22. Cunha MS, da Silva JCF, Nakamoto H, Simão DT, Ferreira MC. Efeito do alopurinol e terapia com oxigênio hiperbárico em modelo de reimplante de membro após isquemia quente em ratos. Rev Col Bras Cir. 2005;32:64-8.

23. Cunha MS. Efeito de inibidores da glicoproteína Ilb-IIla em modelo de amputação subtotal de membro submetido a isquemia quente:estudo experimental em ratos [doutorado]. São Paulo: Faculdade de Medicina, Universidade de São Paulo; 2003.

24. Daniel RK, Kerrigan CL. The omnipotential pig buttock flap. Plast Reconstr Surg. 1982;70: II-5.

25. Kerrigan $\mathrm{CL}$, Zelt RG, Thomson JG. The pig as na experimental animal in plastic surgery research for the study of skin flaps, myocutaneous flaps and fasciocutaneous flaps. Lab Anim Sci. 1986:36:408-11.

26. Hjortdal VE, Hansen ES, Hauge E. Myocutaneous flap ischemia: flow dynamics following venous and arterial obstruction. Plast Reconstr Surg. |992;89: |083-9|.

27. Concannon MJ, Dooley TW, Puckett CL. Improved survival in a replantation model containing ischemic muscle. Microsurgery. 1991; | 2:18-22.

28. Sharma GVRK. Historical overview of antithrombotic and thrombolytic therapy. Am J Med. 1987;83:3-5.

29. ohrich RJ, Handren RN, Kersh R, Hergrueter CA, May JW. Prevention of microvascular thrombosis with short-term infusion of human tissue-type plasminogen activator. Plast Reconstr Surg. 1996;98: | | 8-28.

30. Fukui A, Tamai S. Present status of replantation in Japan. Microsurgery. 1994; I 5:842-7.

3।. Vertos KA, Tsavissis AG. Antithrombotic and antiinflammatory drugs for protection of microvascular anastomosis. Acta Orthop Scand. 1995; 66(Suppl 264):48-9.

32. Atiyeh BS, Hashim HA, Hamdan AM. Recombinant tissue plasminogen activator (rt-PA) thrombolytic therapy in microvascular surgery. Microsurgery. 1999; 19:265-7|.

33. Barry BE, Crapo D. Patterns of accumulation of platelets and neutrophils in rat lungs during exposure to 100\% oxigen and $85 \%$ oxigen. Am Rev Resp Dis. 1985; 132:548-51.

34. Kindwall EP, Gottlieb LJ, Larson DL. Hyperbaric oxygen therapy in plastic surgery: a review article. Plast Reconstr Surg. 1991;88:898908.

35. Edwards RJ, Im MJ, Hoopes JE. Effects of hyperbaric oxigen preservation on rat limb replantation: a preliminary report. Ann Plast Surg. 1991;27:31-5.

Artigo recebido: 20/09/05

Aceito para publicação: 12/09/06 